\title{
Traumatic vertebral artery injury: a review of the screening criteria, imaging spectrum, mimics, and pitfalls
}

\author{
Pranav Sharma ${ }^{1}$, Rahul Hegde ${ }^{1}$, Ashwini Kulkarni ${ }^{2}$, Salil Sharma ${ }^{3}$, Priti Soinn ${ }^{4}$, Puneet S. Kochar ${ }^{1}$, Yogesh Kumar ${ }^{3}$ \\ ${ }^{1}$ Yale New Haven Health Bridgeport Hospital, Connecticut, USA \\ ${ }^{2}$ Teleradiology Solutions, India \\ ${ }^{3}$ Bassett Medical Center, New York, USA \\ ${ }^{4}$ Weil Cornell College Of Medicine, New York, USA
}

\section{Abstract}

\begin{abstract}
Purpose: Traumatic vertebral artery injury (TVAI) can have a varied clinical presentation and appearance on imaging. In this review, we present the screening criteria, spectrum of imaging features, grading, and imaging pitfalls of TVAI. Our review focuses on the imaging of TVAI on computed tomography angiography (CTA), magnetic resonance angiography (MRA), and cases of TVAI mimics.
\end{abstract}

\begin{abstract}
Imaging: The imaging spectrum on CTA can range from either focal or long segment luminal stenosis (the most common findings), smooth or tapered narrowing of lumen, string of pearls appearance, concentric intramural haematoma, intimal flap (the most definite sign), and double lumen of the artery. On time-of-flight MRA, the most common findings include loss of flow void within the vessel due to slow flow, thrombosis or occlusion, and hyperintense signal within the vessel wall due to intramural haematoma on T1 fat-saturated images.
\end{abstract}

Conclusion: The reader should be aware of the screening criteria, common and uncommon findings, variant anatomy, artifacts, and mimics of TVAI when evaluating cases of craniocervical trauma, to be competent in calling in or ruling out injury.

Key words: traumatic vertebral artery injury, vertebral artery dissection, CT angiography, magnetic resonance angiography, digital subtraction angiography.

\section{Introduction}

Traumatic vertebral artery injuries (TVAI) should be suspected clinically in the setting of cervical spine trauma with symptoms of neck pain and/or vertebrobasilar ischaemia. Blunt cerebrovascular injury (BCVI) is the umbrella term used for traumatic vascular injuries of the head and neck arteries; however, we have limited the scope of this article to imaging of TVAI.

The major reasoning for early detection of BCVI is to prevent the possibility of stroke, which can occur in $10-13 \%$ cases, resulting in potential permanent neurologi- cal deficits [1]. With early detection of BCVI, the incidence of stroke can be reduced to as low as $0.5 \%$ by initiation of appropriate treatment, which can include antithrombotic medications or vascular intervention versus untreated BCVI, in which the incidence of stroke can reach $21 \%$ [2].

The clinical scenarios for both BCVI and TVAI are similar. There have been several grading systems and screening criteria set up for detection of BCVI, including the Denver [3], Memphis [4], and Boston [5], criteria based on injury mechanism, injury pattern, and symptoms. The initial screening criteria were set up in the 1990s [6] and were further expanded by the Denver

Correspondence address:

Dr. Pranav Sharma, Yale New Haven Health Bridgeport Hospital, Connecticut, USA, e-mail: drpranavsharma29@gmail.com

Authors' contribution:

A Study design · B Data collection · C Statistical analysis · D Data interpretation · E Manuscript preparation · F Literature search · G Funds collection 
group in 2005 [3]. This modified Denver criteria is the most widely used criteria in practice [1]. More recent literature from 2011 proposed expanding screening criteria to detect asymptomatic early-stage BCVI in order to further reduce the risk of stroke [7]. Application of this expanded screening criteria has improved detection of BCVI from 2.3 to $2.9 \%$ [8] (Table 1 depicts the expanded screening criteria for detection of BCVI proposed in 2011 by Burlew et al. [7]).

However, a balance has to be struck between excess imaging and not missing out on asymptomatic vascular injury. Use of expanded screening criteria or ordering CT angiography (CTA) for all cervical spine fractures can lead to a higher false negative rate. The decision to use either conservative classic screening criteria or more expanded screening criteria remains controversial and an area of ongoing research. However, there remains no doubt that angiographic imaging is mandated for detecting vascular injury in cases of complex cervical spine fractures, subluxations, fractures involving facet joints, or the transverse foramina (covered under the modified Denver criteria), which tend to have a high incidence of concomitant vascular injury and higher yield of vascular injury cases [9]. However, the caveat remains that utilising limited screening criteria can miss up to $20 \%$ of cases. In conclusion, the decision regarding the optimal screening criteria will have to be made by an institution based on its available resources and local standards of care.

\section{Imaging modalities for detection of traumatic vertebral artery injuries}

Digital subtraction angiography (DSA) has historically been considered the gold standard for diagnosis of vascular injury in the head and neck. But DSA is an invasive test, which may not be readily available at smaller hospitals. An invasive test also makes it a less favourable option to be used as a screening tool. DSA is also limited to the evaluation of the vessel lumen and can miss out on detecting cases with non-stenotic intramural haematoma [10]. The role of DSA for diagnosis now would be limited to evaluating high-risk patients who have equivocal or negative results on CTA [11].

Now with improvements in CT technology, CTA has proven to be the initial screening test of choice in head and neck vascular imaging. CTA has recently shown remarkably improved detection of BCVI, especially with 64-slice and higher CT scanners [12]. In one study, CTA demonstrated a high sensitivity of close to $98 \%$ and specificity close to $100 \%$, when compared to DSA [13]. However, achieving these high numbers requires that the radiologists are able to sustain the quality of reading by being more familiar with the spectrum of imaging in BCVI and TVAI, which is the aim and scope of this article.

Doppler Duplex US has a very limited problem-solving role. It cannot be used for screening due to its poor sensitivity because large portions of the vertebral arteries are not accessible for sonographic detection. Hence, its sensitivity for TVAI is even lower than for detection of carotid injuries [14]. The advantages of doppler US, however, are its safety, availability, and ability to detect direction of flow in the vertebral arteries.

Magnetic resonance angiography (MRA) plays a complementary role when combined with MRI for concomitant detection of strokes with MRI and vascular injury with MRA. However, when judged by its ability to detect vascular injury, it is less sensitive compared to either CTA or DSA. Studies have found low sensitivities for MRA for detection of TVAI in the range of $47-60 \%$ [4].

Table 1. The 2011 expanded screening criteria for detecting blunt cerebrovascular injury (BCVI) (adapted from [7])

\begin{tabular}{|c|c|}
\hline Signs/symptoms of BCVI & Risk factors for BCVI \\
\hline Arterial haemorrhage (from neck, nose, or mouth) & High-energy transfer mechanism associated with: \\
\hline Cervical bruit (in younger than 50 years of age) & Facial fracture of LeFort II or III type \\
\hline Expanding cervical haematoma & Mandible fracture \\
\hline $\begin{array}{l}\text { Focal neurologic defect: transient ischemic attack, hemiparesis, } \\
\text { vertebrobasilar symptoms, Horner syndrome }\end{array}$ & Complex skull fracture/basilar skull fracture/occipital condyle fracture \\
\hline Neurologic deficit inconsistent with head computed tomography & Closed head injury consistent with DAl and GCS $<6$ \\
\hline \multirow[t]{7}{*}{ Stroke on computed tomography or magnetic resonance imaging } & $\begin{array}{l}\text { Cervical vertebral body or transverse foramen fracture, subluxation, } \\
\text { or ligamentous injury at any level; any fracture at C1-C } 3\end{array}$ \\
\hline & Near hanging with anoxic brain injury \\
\hline & $\begin{array}{l}\text { Clothesline-type injury or seat belt abrasion with significant swelling, pain, } \\
\text { or altered mental status* }\end{array}$ \\
\hline & Traumatic brain injury with thoracic injuries* \\
\hline & Scalp degloving* \\
\hline & Thoracic vascular injuries* \\
\hline & Blunt cardiac rupture* \\
\hline
\end{tabular}

\footnotetext{
* - signifies new additions to the 2011 expanded screening criteria by Burlew et al. when compared to the previous 2005 Denver screening criteria
} 
We present a brief overview of normal and variant anatomy of the vertebral arteries. We follow that with a pictorial review of the imaging spectrum of TVAI including grading of the injury along with the mimics and pitfalls to be aware of.

\section{Anatomy}

\section{Normal anatomy}

The vertebral artery (VA), in most people, are paired structures arising from the first part of the subclavian arteries. It then ascends posterior to internal carotid artery, obliquely superiorly and medially towards the cervical spine. Either at the $\mathrm{C} 6$ or $\mathrm{C} 7$ level, it enters the foramen transversarium/ transverse foramen, which is an aperture within the transverse processes of the cervical vertebrae. It ascends through the foramen transversaria till $\mathrm{C} 2$. The transverse foramina contain VA, vertebral veins, and sympathetic plexus. From C2, the VA sweeps laterally to pass through the transverse foramen of $\mathrm{C} 1$ and passes superiorly to pierce the dura and arachnoid to extend intracranially and then joins the contralateral VA to form the basilar artery. Anatomical landmarks have been utilised to divide the vertebral artery into segments the V1 (extraosseous segment) from origin to entry into its caudal most transverse foramen, V2 (foraminal segment or pars transversaria) - typically from C6 to C2 within the transverse foramina, V3 (extraspinal segment or atlas loop) from C2 to dura at the skull base, and V4 (intradural intracranial segment) from dura to confluence (Figure 1).

\section{Variant anatomy}

The VA may arise directly from the brachiocephalic arteries or directly from the aortic arch instead of the subclavian artery. The VA may be asymmetric in size with one vessel being dominant. The left VA is more commonly dominant. The finding of VA hypoplasia, although a developmental variant, has been reported to have a higher incidence of VA dissection and posterior circulation stroke [15]. It can sometimes be difficult to conclude whether smaller size of the VA is due to developmental hypoplasia or due to acquired stenosis. Hypoplasia is usually a diffuse finding and the entire VA is diffusely small calibre. However, there may be, on occasions, an isolated narrow V4 segment after the origin of the posterior inferior cerebellar artery or even the V4 segment terminating as the posterior inferior cerebellar artery [16]. These should not be mistaken for stenosis or TVAI. A useful clue for developmental hypoplasia is that the size of the transverse foramen in also small with a developmentally small VA. Stenoses tend to occur segmentally whereas hypoplasia is a diffuse finding. Duplication and fenestration of the vertebral arteries and of the vertebra-basilar junction is also a rare variant $[17,18]$. This should be identified as a variant and not mistaken for the two-vessel sign of TVAI.

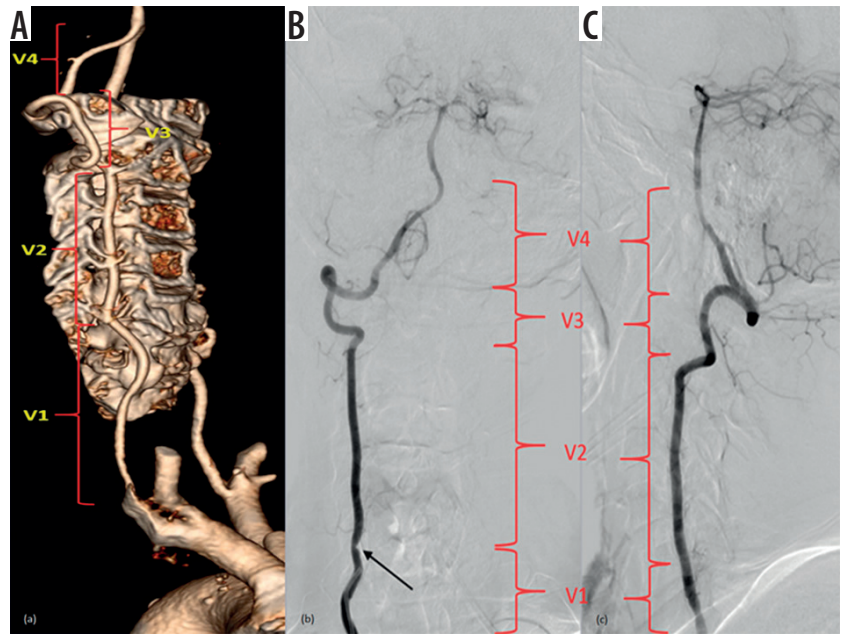

Figure 1. Three-dimensional (3D), volume-rendered (A), and digital subtraction angiography images (B-C) show the vertebral artery segments. $\mathrm{V} 1$ - between vertebral artery origin and its entry into the transverse foramen of the $(6$ vertebra, $\mathrm{V} 2$ - foraminal course between the transverse processes of $\mathrm{C} 6$ to $\mathrm{C} 2, \mathrm{~V} 3$ - atlas loop region, and $\mathrm{V} 4$ - intracranial segment. Note there is luminal narrowing in V1 segment in image (B) (black arrow)

\section{Mechanism and grades of traumatic vertebral artery injuries}

The mechanism of TVAI usually involves both pre-existing intrinsic susceptibility and a precipitating event. Although a normal VA can undergo injury from major trauma, minor trauma can also cause TVAI when there is an underlying arteriopathy, which may be congenital or genetic, like connective tissue disorders, fibromuscular dysplasia, or vessel wall weakening caused by infection or inflammation of the vessel wall. Pathological evaluation of cases of spontaneous cervical artery dissections have found changes of Ehlers Danlos syndrome and Marfan syndrome in the vessel wall [19]. This, superimposed with a minor or major trauma, leads to intimal tears, which are the subtlest form of injury. These tears can be seen as thin intimal flaps on imaging in the vessel lumen. This dissection can progress further when luminal blood enters through the intimal defect and extravasates in the vessel wall creating a false lumen. The false lumen can extend for a variable length and can remain patent or more frequently thrombose due to slower flow. More severe injury can manifest when the vessel wall ruptures, and a pseudoaneurysm may be formed if the rent in the vessel wall is small. In most severe forms of injury, the vessel may become occluded or transacted, which is usually fatal [20-22].

On review of the literature, the predominant aetiology was found to be high-velocity motor vehicle-related injuries; however, other cause like a fall, sporting accident, chiropractic manipulation, and hanging, although less common, have been described [9]. Distractive flexion injury is the main mechanism for TVAI, instead of the hyperextension injury as previously thought $[23,24]$. 
Table 2. Denver grading of severity of blunt cerebrovascular injury (BCVI) (from [3])

\begin{tabular}{|ll|}
\hline Grade I & $\begin{array}{l}\text { Irregularity of vessel wall or dissection/ } \\
\text { intramural haematoma with }<25 \% \text { stenosis }\end{array}$ \\
\hline Grade II & $\begin{array}{l}\text { Intramural thrombus or raised Intimal flap or dissection/ } \\
\text { intramural haematoma with }>25 \% \text { stenosis }\end{array}$ \\
\hline Grade III & Pseudoaneurysm \\
\hline Grade IV & Vessel occlusion \\
\hline Grade V & Vessel transaction \\
\hline
\end{tabular}

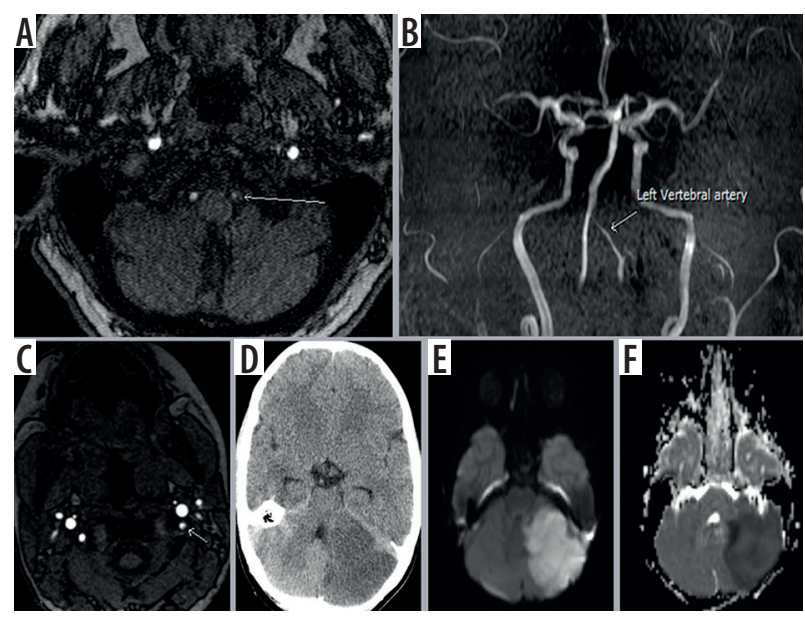

Figure 2. Axial non-contrast time-of-flight (TOF) magnetic resonance angiography (MRA) image $(A)$ and reformatted $3 \mathrm{D}$ maximum intensity projection image (B) showing smooth tapered narrowing of the left vertebral artery consistent with grade II traumatic vertebral artery injury (TVAl) (white arrow). Another patient's axial TOF MRA image (C) showing irregularity of lumen of left vertebral artery suggesting focal dissection (grade ITVAI) and the same patient's axial computed tomography (D), diffusion weighted image (E), and $A D C$ map (F) of the brain showing acute left cerebellar infarct

Location of TVAI: Most TVAI are located in the V2 or V3 segment $[25,26]$. The V2 segment is the most commonly affected in adult TVAI. In infants and children, the V3 and upper V2 segment are more commonly affected [27].

The grading of TVAI severity proposed by the Denver group is the most widely used and is depicted in Table 2 [9]. We subsequently present examples of these grades in the imaging spectrum that follows.

\section{Imaging spectrum}

\section{Computed tomography angiography}

Technique: CT angiography (CTA) of head and neck, when performed with modern 64-slice or higher CT scanner, provides thin-slice ( $1 \mathrm{~mm}$ or less), high-resolution imaging of the arterial lumen and the wall of the VA. Bolus tracking of the contrast is incorporated in the protocol in order to obtain optimal phase of maximum opacification of the arteries. Modern CT scanners are faster at scanning as well limiting late contrast bolus and artefacts from thicker slices associated with older CT scanners. Generation of multiplanar coronal and sagittal reconstructions, MIP (maximum intensity projection), and VR (volume rendered) imaging are among the common protocols for CT angiographic imaging, and these multiple views and projections help to establish a more confident assessment of the anatomy and pathology.

CTA Findings of TVAI: The findings on CTA will vary as per the grade of injury. The commonly encountered imaging findings of TVAI on CTA are smooth tapered luminal narrowing, concentric or focal luminal narrowing of the VA (Figure 2A-C), or total occlusion (Figure 3), with these findings seen in more than $50 \%$ cases. Other findings seen are, double lumen (Figure 4A), intimal flap (Figure 4B-G), dilatation of artery (Figure 4F-H), beaded appearance of artery or string of pearls appearance (Figure $4 \mathrm{I}-\mathrm{K}$ ) or stenosis of lumen. The most definite sign is double lumen or intimal flap, which is not frequently seen (in around $22 \%$ cases), and the most common imaging characteristic is nonspecific luminal stenosis (seen in more than $50 \%$ cases) [28]. CTA can show contrast extravasation or pseudoaneurysm formation, which are signs of more severe injury (Figure 5).

CTA Pitfalls: The CTA images may be affected by poor bolus timing, bone, dental, or metallic artefacts in the spine or from patient's motion, swallowing, or pulsation artefacts, which limit image interpretation. Poor bolus may show contrast in arteries and veins simultaneously, giving the appearance of two lumens. Tortuosity of the vessel may also give the false appearance of two lumens. Atheroscle-
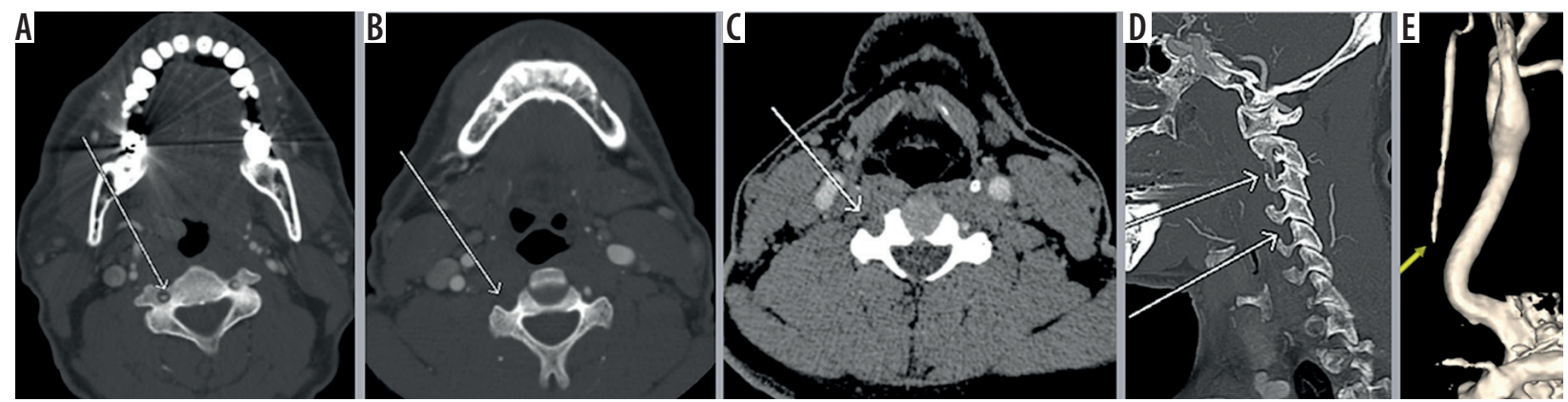

Figure 3. Axial contiguous computed tomography images (A-C) showing total occlusion of the right vertebral artery. The reformatted image (D) shows opacification of the upper portion of the vertebral artery, probably from retrograde filling of the lumen (white arrow). Three-dimensional volume-rendered image (E) showing occlusion of the V1 segment of the vertebral artery (yellow arrow) 

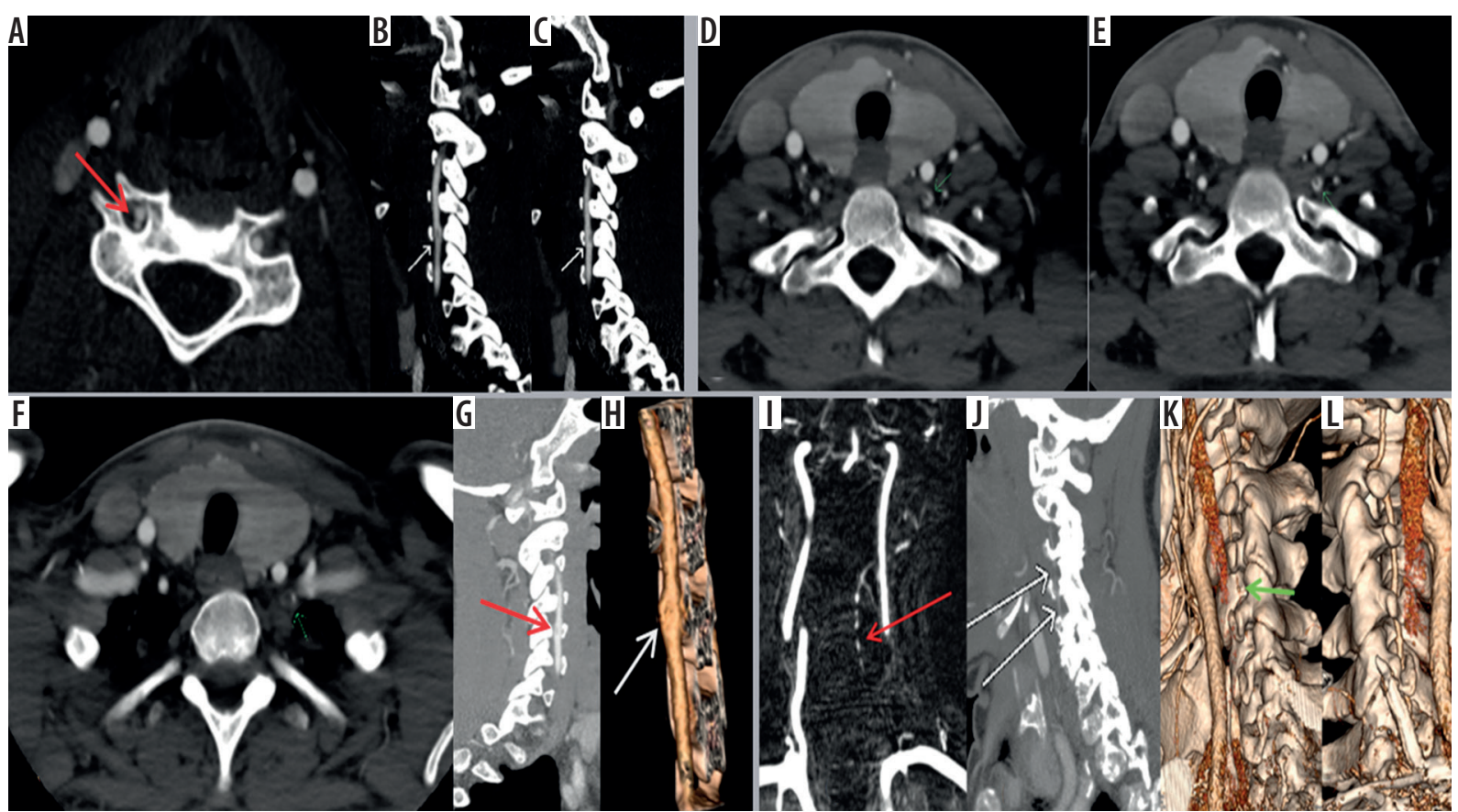

Figure 4. Axial computed tomography angiography (CTA) image (A) showing double lumen in right vertebral artery s/o dissection (grade II traumatic vertebral artery injury [TVAI]). Multiplanar reformatted image (B-C) showing the intimal flap (grade II). Contiguous slices of axial CTA images (D-E) of another patient showing intimal flap in left vertebral artery (green arrow). Axial CTA image (F) showing increase in luminal diameter of the left vertebral artery (green arrow). The reformatted image (G) in same patient showing the dilatation (white arrow) of the artery with intimal flap in the lumen (red arrow) and three-dimensional (3D) volume-rendered image $(\mathrm{H})$ showing abrupt expansion of the artery (white arrow). Another patient's coronal magnetic resonance angiography (MRA) image (I) showing beaded narrowing of the left vertebral artery (red arrow) and the reformatted image (J) showing the irregular filling of the left vertebral artery (white arrow). The three-dimensional (3D) volume-rendered image (K) in the same patient showing beaded appearance of left vertebral artery "string of pearls appearance" (green arrow). Comparative normal appearance of contralateral vertebral artery on the three-dimensional (3D) volume-rendered image (L)

rotic plaque or ulcerated plaques may have an appearance of intimal flap with contrast in vessel the lumen and in the ulceration outside the expected location of the arterial wall, and lead to false positive interpretation of TVAI. This is an issue with evaluation in older patients who have pre-existing atherosclerotic changes in their vessels. Arterial wall thickening or diminished flow due to severe stenosis may cause false positive interpretation for TVAI [29]. Developmentally hypoplastic VA can mimic long segment arterial injury, but usually can be ascertained by a smaller ipsilateral transverse foraminal diameter (Figure 6). Another limitation is that CTA is not a dynamic study, so it is difficult to interpret if there is retrograde filling of the affected vertebral artery or reversal of flow. Refer to Table 3 for a summary of the TVAI CTA findings and pitfalls.

Care should be taken while calling in a TVAI because there could be other medical conditions that can lead to similar findings of occlusion or stenosis. The patient's clinical history should be kept in mind. The most common mimickers are fibromuscular dysplasia, atherosclerosis, and post-radiation treatment changes.

\section{Magnetic resonance angiography and magnetic resonance imaging}

Technique: TVAI can be assessed with MRA either with or without contrast. The ability to image without contrast is especially helpful in patients with contraindications to iodinated contrast material. The imaging protocol for non-contrast MRA includes either 2D or 3D time-offlight imaging, which detects the velocity of flowing protons. Another technique is phase contrast imaging, which can provide quantitative estimation of the flow velocity. T1 images of the neck with fat saturation are also performed as part of standard protocol because the blood in the false lumen - the intramural haematoma - appears hyperintense in the subacute phase (Figure 7). Usage of gadolinium contrast is optional, but it does improve the imaging of the lumen of the arteries secondary to opacification by $\mathrm{T} 1$ relaxation effects of gadolinium. MRI of the brain can be performed at the same time as the MRA. The MRI, especially diffusion sequence, can help detect the presence of acute infarction, for which it is exquisitely sensitive and specific when compared to CT. Other factors that affect the imaging characteristics are the field strength of the scanner, patient body habitus, the signal intensity from the surrounding structures, and the imaging sequence protocol used $[30,31]$.

\section{Magnetic resonance angiography findings of traumatic vertebral artery injuries}

The intramural haematoma in the false lumen after dissection is hyperintense on T1-weighted images in subacute 


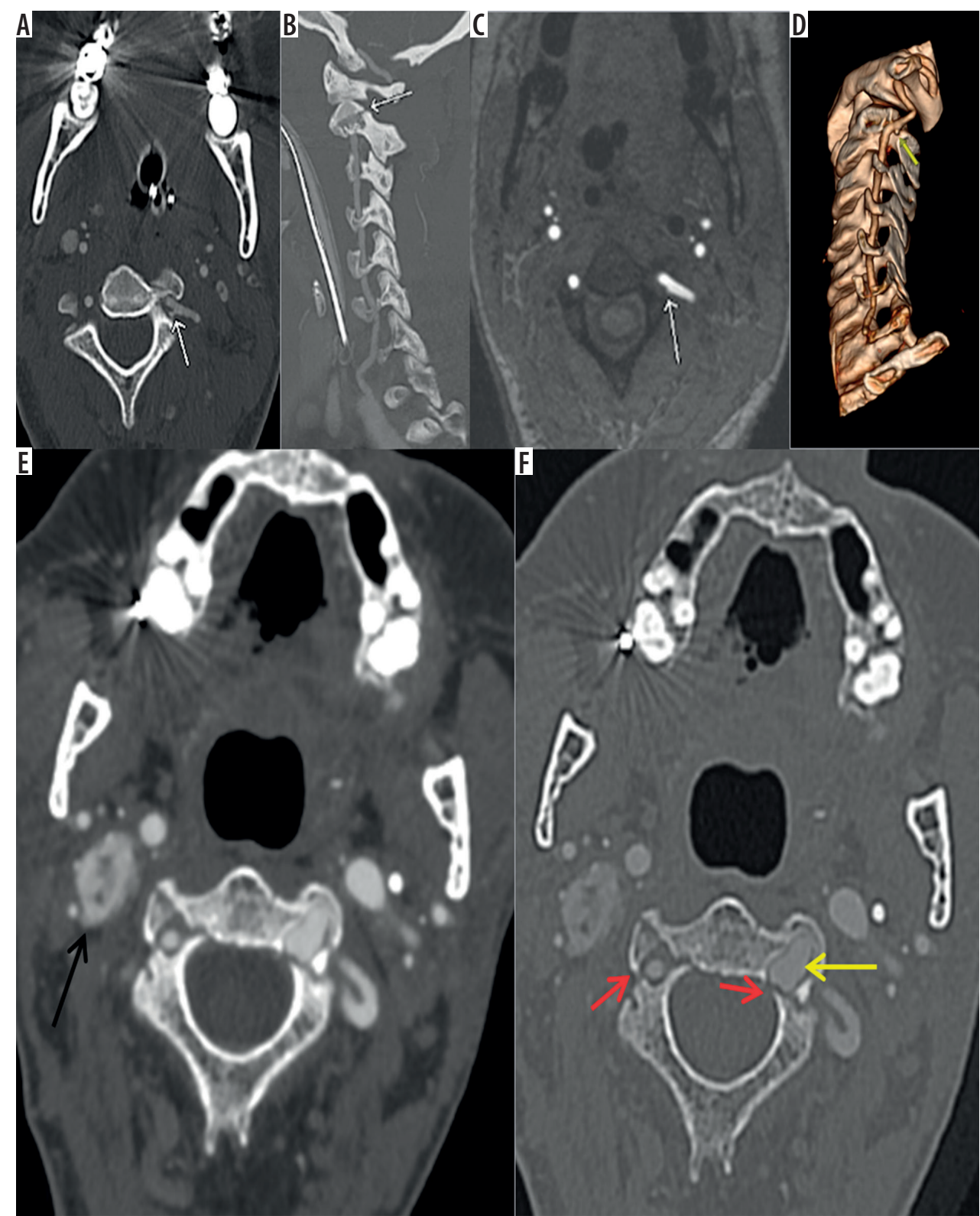

Figure 5. Axial computed tomography angiography (CTA) image (A) showing contrast outpouching from the V3 segment of the left vertebral artery (white arrow). The reformatted image (B) shows the fracture through the transverse foramen (white arrow). The axial magnetic resonance image (C) shows the small signal outpouching from the same site as was depicted on CTA (white arrow) and the three-dimensional (3D) volume-rendered image (D) showing the pseudoaneurysm clearly. CTA images (E, F) showing left pseudoaneurysm (yellow arrow) with fractures through the foramen transversarium (red arrows); also note incidental detection of a hypervascular right glomus tumour adjacent to cervical internal carotid artery (black arrow)

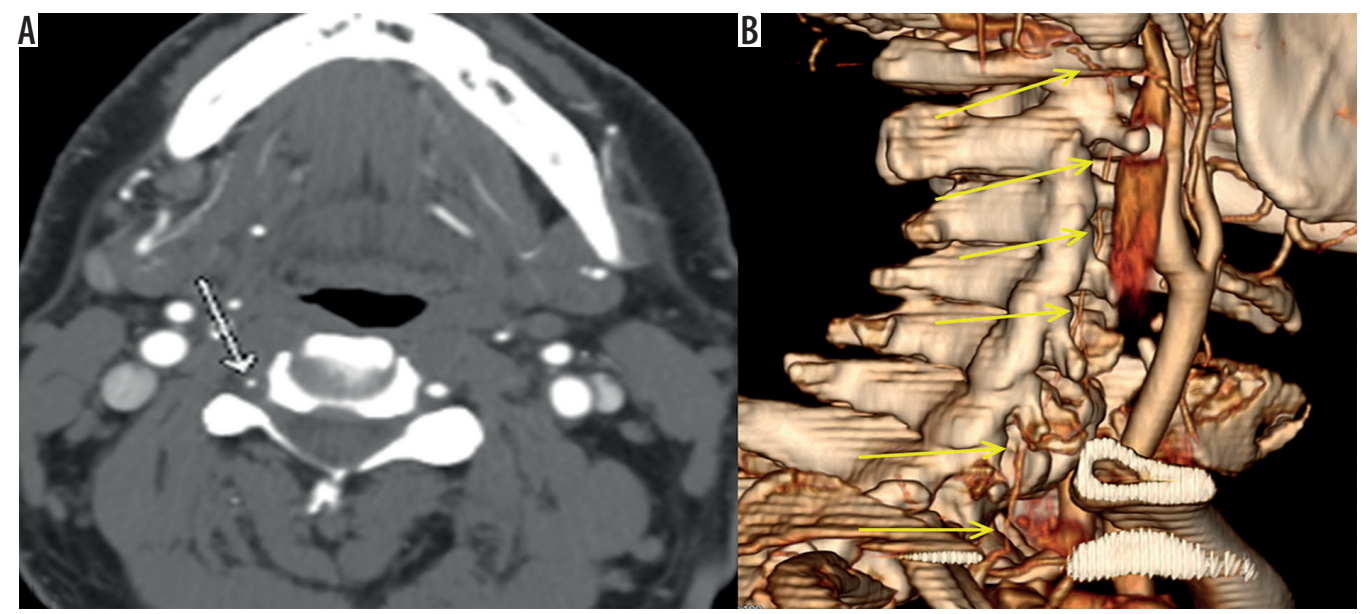

Figure 6. Axial computed tomography angiography image (A) showing asymmetry of lumen of the right vertebral artery. Three-dimensional (3D) volumerendered image (B) shows that the whole of the right vertebral artery is hypoplastic (yellow arrows) 
Table 3. Findings and pitfalls of traumatic vertebral artery injury on computed tomography angiography

\begin{tabular}{|l|l|}
\hline Findings & \multicolumn{1}{c|}{ CTA pitfalls } \\
\hline Concentric or focal luminal narrowing or stenosis & Poor bolus timing \\
\hline Smooth tapered lumen & Bone, dental, or metallic artefacts \\
\hline Intimal flap & Motion due to patient movement, swallowing, pulsation \\
\hline Double lumen & Atherosclerotic or ulcerated plaques in vessel wall with contrast in plaque - false positive \\
\hline Dilatation of artery & Diminished flow or wall thickening giving appearance of double lumen - false positive \\
\hline String of pearls & Asymmetric VA diameter or hypoplastic VA - false positive \\
\hline Total occlusion & Steal phenomenon not evaluated as not a dynamic study \\
\hline
\end{tabular}
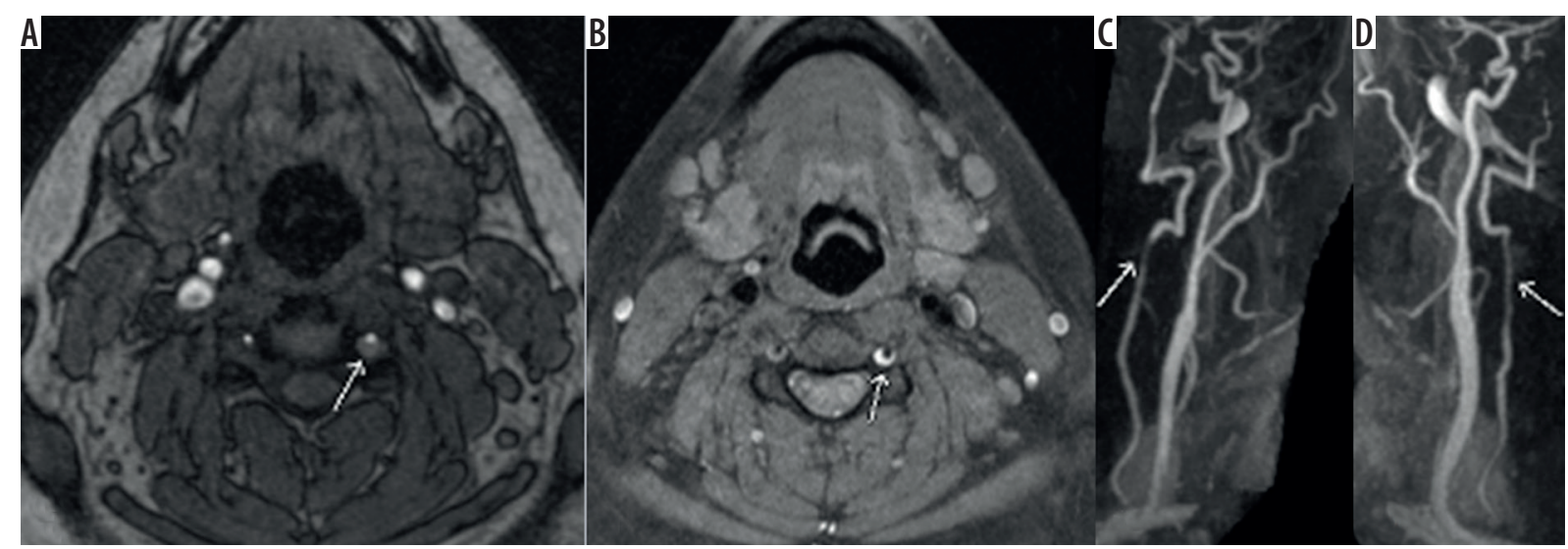

Figure 7. Axial time-of-flight magnetic resonance angiography image (A) showing intramural haematoma as seen by abnormal signal surrounding and narrowing the left vertebral artery flow signal (white arrow). T1 fat-saturated image (B) showing the T1 hyperintense signal consistent with intramural haematoma (white arrow) and the reformatted images showing luminal narrowing (C, D) (white arrow)

stages between seven days and two months and is detected on the fat-saturated imaging. Subacute haematoma is seen as crescentic hyperintense areas around an eccentric flow void [30,32]. However, it is isointense in acute or chronic stages (within six months). When the haematoma is isointense, as in acute or chronic dissections, it becomes tough to detect. Other findings seen on MRA are loss of flow void or occlusion (Figure $8 \mathrm{~A}-\mathrm{B}$ ), similar to what can be seen on CTA. The 3D TOF technique is better than the 2D TOF in regard to spatial resolution, but it increases the scan time. Contrast MRA has greater ability to evaluate the lumen, especially for subtle luminal irregularities and stenosis [32,33].

MRA Pitfalls: The false positives on MRA are due to turbulent flow producing hyperintense signal in the periphery with central flow void simulating stenosis or intramural haematoma (Figure 8C-D). Additionally, periarterial structures like fat or veins can simulate intramural haematoma or pseudoaneurysm (Figure $8 \mathrm{E}-\mathrm{F}$ ). These are more confounding when there is developmental asymmetry in the size of the VA (Figure 8F). Comparison with other sequences, fat saturation, or CT images may be helpful in such cases $[29,34]$. Entry slice phenomenon causing a bright signal in the artery at the extreme of the imaging volume due to entry of unsaturated spin can simulate an intramural abnormal signal or the absence of an expect- ed flow void simulating dissection. Also, the flow-related enhancement in the veins adjacent to VA can simulate intramural haematoma or dissection on unenhanced MRA (TOF). These can be overcome by the application of saturation pulses cranially and caudally to the imaging volume $[29,35]$. V1 segment VA dissection may be missed with V1 inadvertently not included in the image volume, and small dissection may be missed due to gaps in image slabs [31]. In early or acute stages, the intramural haematoma may be isointense on T1-weighted images, in such cases other signs may be helpful, like luminal narrowing or pseudoaneurysm formation. In subacute stages, the bright intramural haematoma may be masked by the background bright signal from fat or indistinguishable from the flow-related enhancement on MRA. The bright signal from an occluded vessel may obscure the bright intramural haematoma; however, this can be resolved by contrast-enhanced MRA with subtraction of unenhanced images [29]. Asymmetry in the size of the VA may be mistaken for dissection in a smaller lumen artery (Figure 8F). Careful observation of the periarterial bright signal on TOF, only seen in dissection rather than normal artery, should be performed [34]. Comparing the luminal diameter on the VA above and below the site of injury can help in calling in or ruling out normal asymmetry with confidence. In contrast-enhanced MRA there might be segmental blurring of the signal in 

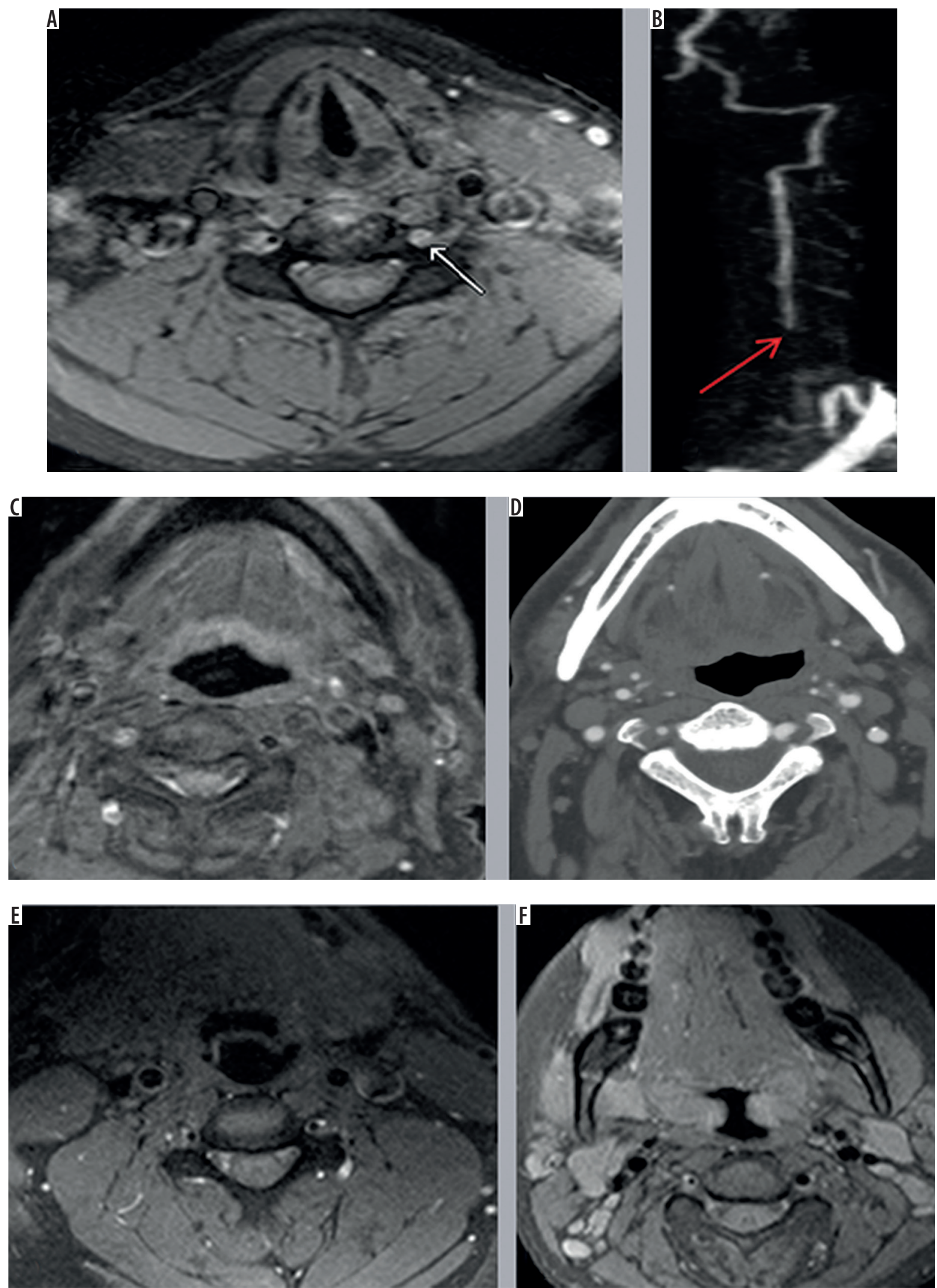

Figure 8. Axial T1 fat-saturated magnetic resonance (MR) image (A) showing loss of flow void in left vertebral artery and abnormal hyperintense signal around it s/o dissection with intramural haemorrhage (white arrow). Reformatted time-of-flight magnetic resonance angiography image (B) shows complete occlusion of the left vertebral artery (red arrow) with distal reconstitution. Another patient with axial T1 fat-saturated MR image (C) showing hyperintense signal in right vertebral artery with loss of flow void, and the computed tomography angiography (D) performed the same day showed contrast filling of the right vertebral artery; thus, it was concluded that the abnormal signal is secondary to the flow artefact. Other patient axial MR images (E, F) showing venous contamination producing hyperintense signal along the periphery of bilateral vertebral arteries. Note asymmetry in the size of the vertebral arteries, with left being dominant 
Table 4. Findings and pitfalls of traumatic vertebral artery injury on magnetic resonance angiography (MRA)

\begin{tabular}{|c|c|}
\hline Findings & MRA pitfalls \\
\hline $\begin{array}{l}\text { Intramural haematoma - signal intensity depends on the age } \\
\text { of haematoma }\end{array}$ & $\begin{array}{l}\text { Hyperintense peripheral signal with flow void centrally due to turbulent flow - } \\
\text { false positive }\end{array}$ \\
\hline Loss of flow void or occlusion & Periarterial fat, haematoma - false positive \\
\hline Contrast shows vessel abnormalities & Asymmetric or hypoplastic vertebral artery - false positive \\
\hline \multirow[t]{7}{*}{ Steal phenomenon and retrograde filling can be assessed } & Entry slice phenomenon causing bright signal - false positive \\
\hline & Flow related enhancement in the veins adjacent to vertebral artery - false positive \\
\hline & V1 not included in field of view - false negative \\
\hline & Small dissection in gaps in image slab - false negative \\
\hline & $\begin{array}{l}\text { Bright intramural haematoma merging with bright signal from adjacent far, } \\
\text { haematoma or occluded vessel - false negative }\end{array}$ \\
\hline & Feathering artefact - false positive \\
\hline & Pseudoaneurysm missed at skull base \\
\hline
\end{tabular}
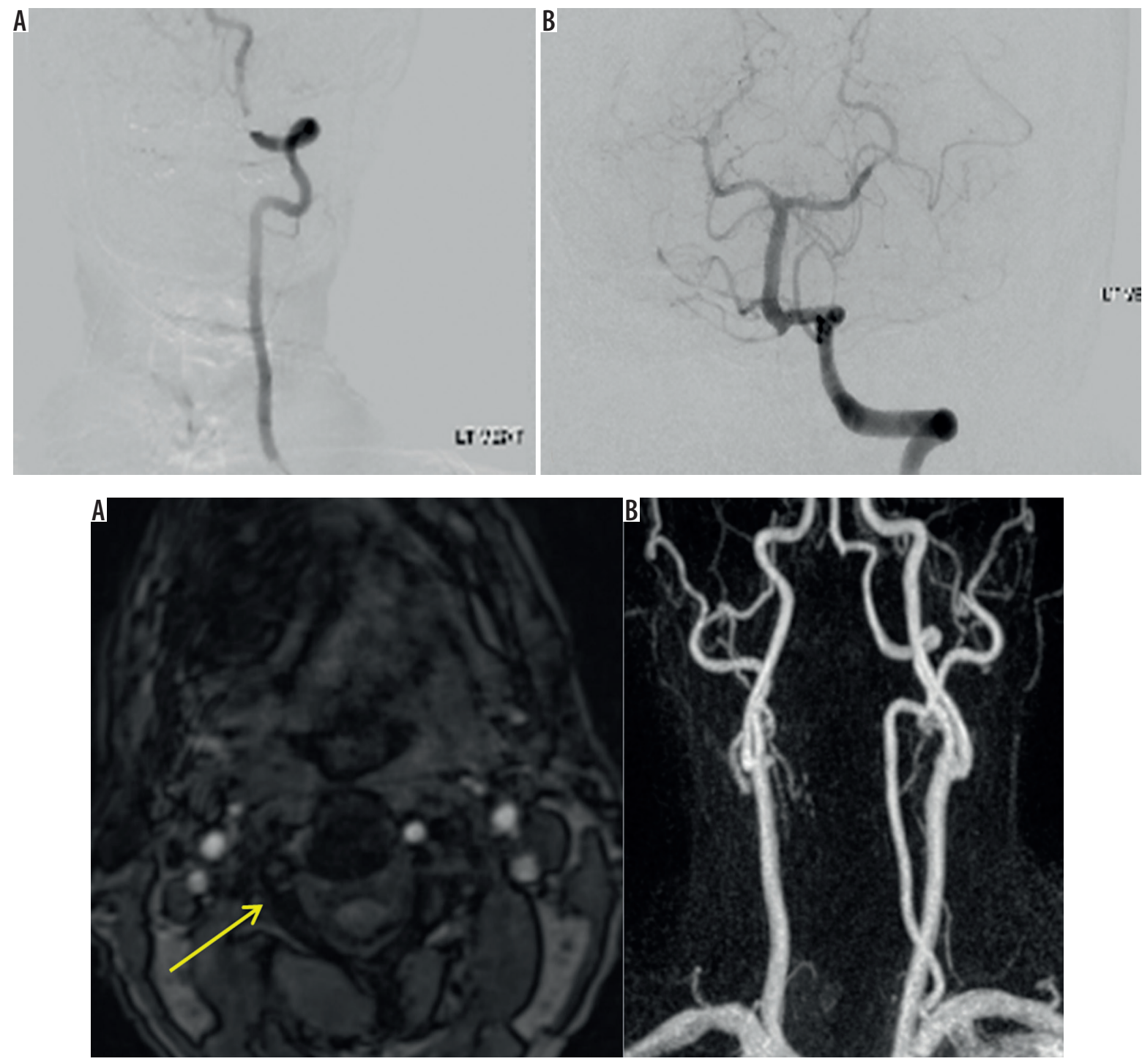

Figure 9. Digital subtraction angiography images (A, B) showing complete occlusion of right vertebral artery without reconstitution by collaterals. Timeof-flight magnetic resonance angiography image (C) showing absence of flow signal in the right vertebral artery (yellow arrow). Reformatted image (D) confirming the same with absent signal of right vertebral artery 

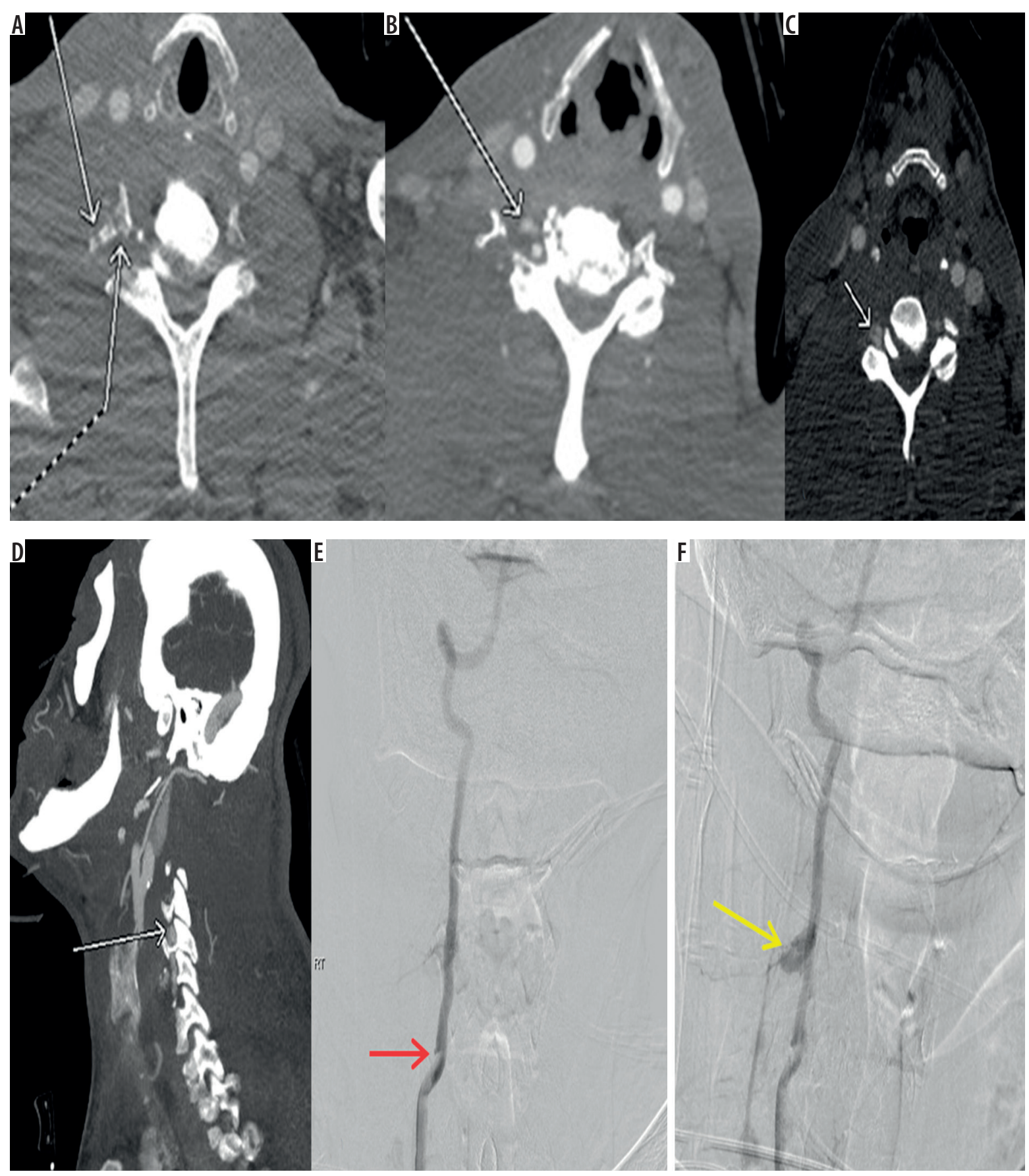

Figure 10. Computed tomography angiography (CTA) image (A) showing the factures through the transverse foramen (white solid arrow) with irregularity of the right vertebral artery (white arrow with dotted line). CTA Image (B, C) showing mild dilatation of the right vertebral artery with intraluminal flap (grade Il injury) (white arrow). Follow-up reformatted image (D) showing pseudoaneurysm formation in right vertebral artery (white arrow). Angiographic images (E) demonstrating the dissection with intimal flap (red arrow) and pseudoaneurysm (yellow arrow) (F). Patient had good collateral circulation without enlargement of pseudoaneurysm and so was managed conservatively

VA due to rapid circulation times, especially in young patients, called the feathering artefact, and it may simulate small stenosis. This can be overcome by using an earlier trigger time with elliptical centric contrast enhanced MRA [33]. MR is also limited in the detection of pseudoaneurysms because they are mostly seen at the skull base with abnormal flow or signal within and may mimic a mass lesion $[34,36]$. Refer to Table 4 for a summary of the TVAI MRA findings and pitfalls.

\section{Digital subtraction angiography}

DSA, although traditionally considered a gold standard, is limited by lack of ability to assess the arterial wall configuration and thickness. The common signs seen on DSA are: complete occlusion of lumen (Figure 9), "string sign", and "string and pearl sign", which refers to long tapered eccentric irregular stenosis without or with distal dilatation. Pathognomonic signs, such as intimal flap and or 
double lumen, are rarely observed [37]. When there is a tapered occlusion, it is referred to as the "Flame Sign" [38]. The major advantage of DSA is that it is a dynamic acquisition. Hence it does not suffer as much from contrast kinetics and bolus timing as CTA. Hence, DSA is sometimes performed to supplement evaluation in indeterminate cases, assess the collateral circulation, or rarely as part of endovascular repair. If there is good collateral circulation, patients usually do well with conservative management (Figure 10).

\section{Doppler duplex ultrasound}

US doppler duplex imaging has limited sensitivity in detecting mainly extracranial VA dissection. Distal V2 and V3 segments cannot be adequately evaluated due to a poor acoustic window. However, there are some features that are beneficial, like rapid mobility, cost-effectiveness, and non-invasiveness, but it is not sensitive enough to exclude intimal tears [39]. Hence, it is not suited to initial evaluation of a trauma patient when injury needs to be ruled out. However, it can be useful as a problem-solving tool. The ability to easily detect direction of flow in the vertebral artery is also helpful when compared to CTA and MRA. Spectral imaging with peak systolic velocity values and waveform morphology helps estimate the presence of stenosis. If TVAI can be visualised accurately, then it has a role for follow-up of vertebral artery dissection [40].

\section{Management and treatment}

TVAIs are usually managed conservatively with either anticoagulation or antiplatelet therapy. The used anticoagulant medications include warfarin and heparin. Used antiplatelet agents include aspirin, clopidogrel, and dipyridamole. The optimal regimen with respect to agent, duration of treatment, or end point of therapy is not known. Often the associated injuries limit antithrombotic therapy. In such cases, endovascular interventions are another therapeutic option [41]. Grade I and II injuries are managed conservatively with medical management. Anticoagulation, typically with heparin, acutely followed by three months of warfarin was the traditional management. In 2015, the Cervical Artery Dissection in Stroke Study trial (CADISS) suggested no difference in efficacy of antiplatelet and antithrombotic agents in preventing stroke and deaths in patients with symptomatic vertebral artery dissection [42]. This may encourage wider use of antiplatelet agents over anticoagulation agents considering the better safety profile of the former with respect to haemorrhagic complications. For grade III injuries with pseudoaneurysm, the initial treatment again is antithrombotic therapy. However, if the size of the pseudoaneurysm reaches from 1-1.5 cm or is symptomatic then endovascular stenting or coiling may be performed [41,43]. For grade IV injuries with arterial thrombosis, the initial treatment is with antithrombotic therapy, and treatment outcomes with endovascular therapy are not known yet. Grade V injuries are mostly fatal, and surgical exploration is recommended if possible.

\section{Conclusions}

Imaging with CTA is the initial test of choice in patients with trauma, who meet the screening criteria for the possibility of cervical vascular injury. MRA, DSA, and Doppler duplex US have complementary secondary roles in imaging. Early detection is key because initiation of therapy can dramatically reduce the incidence of associated strokes. Familiarity with the key findings of TVAI, anatomic variations, mimics, and pitfalls depicted and summarised in this review should assist the readers in more accurate estimation of TVAI.

\section{Conflict of interest}

The authors report no conflict of interest.

\section{References}

1. Nagpal P, Policeni BA, Bathla G, et al. Blunt Cerebrovascular Injuries: Advances in Screening, Imaging, and Management Trends. Am J Neuroradiol 2018; 39: 406-414.

2. Cothren CC, Biffl WL, Moore EE, et al. Treatment for blunt cerebrovascular injuries: equivalence of anticoagulation and antiplatelet agents. Arch Surg 2009; 144: 685-690.

3. Cothren CC, Moore EE, Ray CE Jr, et al. Cervical spine fracture patterns mandating screening to rule out blunt cerebrovascular injury. Surgery 2007; 141: 76-82.

4. Miller PR, Fabian TC, Croce MA, et al. Prospective screening for blunt cerebrovascular injuries: analysis of diagnostic modalities and outcomes. Ann Surg 2002; 236: 386-393; discussion 93-95.
5. Buch K, Nguyen T, Mahoney E, et al. Association between cervical spine and skull-base fractures and blunt cerebrovascular injury. Eur Radiol 2016; 26: 524-531.

6. Biffl WL, Moore EE, Offner PJ, et al. Optimizing screening for blunt cerebrovascular injuries. Am J Surg 1999; 178: 517-522.

7. Burlew CC, Biffl WL, Moore EE, et al. Blunt cerebrovascular injuries: redefining screening criteria in the era of noninvasive diagnosis. J Trauma Acute Care Surg 2012; 72: 330-335; discussion 6-7, quiz 539.

8. Geddes AE, Burlew CC, Wagenaar AE, et al. Expanded screening criteria for blunt cerebrovascular injury: a bigger impact than anticipated. Am J Surg 2016; 212: 1167-1174. 
9. Cothren CC, Moore EE, Biffl WL, et al. Cervical spine fracture patterns predictive of blunt vertebral artery injury. J Trauma 2003; 55: 811-813.

10. Lum C, Chakraborty S, Schlossmacher M, et al. Vertebral artery dissection with a normal-appearing lumen at multisection CT angiography: the importance of identifying wall hematoma. AJNR Am J Neuroradiol 2009; 30: 787-792.

11. Rutman AM, Vranic JE, Mossa-Basha M. Imaging and management of blunt cerebrovascular injury. Radiographics 2018; 38: 542-563.

12. Paulus EM, Fabian TC, Savage SA, et al. Blunt cerebrovascular injury screening with 64-channel multidetector computed tomography: more slices finally cut it. J Trauma Acute Care Surg 2014; 76: 279283; discussion 84-85.

13. Eastman AL, Chason DP, Perez CL, et al. Computed tomographic angiography for the diagnosis of blunt cervical vascular injury: is it ready for primetime? J Trauma 2006; 60: 925-929; discussion 9.

14. Bromberg WJ, Collier BC, Diebel LN, et al. Blunt cerebrovascular injury practice management guidelines: the Eastern Association for the Surgery of Trauma. J Trauma 2010; 68: 471-477.

15. Zibis A, Mitrousias V, Galanakis N, et al. Variations of transverse foramina in cervical vertebrae: what happens to the vertebral artery? Eur Spine J 2018; 27: 1278-1285.

16. Liu IW, Ho BL, Chen CF, et al. Vertebral artery terminating in posterior inferior cerebellar artery: A normal variation with clinical significance. PLoS One 2017; 12: e0175264.

17. Ginsberg LE. Atlas of Neuroradiologic Embryology, Anatomy, and Variants. Am J Roentgenol 2001; 176: 48.

18. Satti SR, Cerniglia CA, Koenigsberg RA. Cervical vertebral artery variations: an anatomic study. AJNR Am J Neuroradiol 2007; 28 : 976-980.

19. Brandt T, Morcher M, Hausser I. Association of cervical artery dissection with connective tissue abnormalities in skin and arteries. Front Neurol Neurosci 2005; 20: 16-29.

20. Fassett DR, Dailey AT, Vaccaro AR. Vertebral artery injuries associated with cervical spine injuries: a review of the literature. J Spinal Disord Tech 2008; 21: 252-258.

21. Debette S, Leys D. Cervical-artery dissections: predisposing factors, diagnosis, and outcome. Lancet Neurol 2009; 8: 668-678.

22. Schievink WI, Debette S. Etiology of cervical artery dissections: the writing is in the wall. Neurology 2011; 76: 1452-1453.

23. Vaccaro AR, Klein GR, Flanders AE, et al. Long-term evaluation of vertebral artery injuries following cervical spine trauma using magnetic resonance angiography. Spine (Phila Pa 1976) 1998; 23 : 789-794; discussion 95.

24. Veras LM, Pedraza-Gutierrez S, Castellanos J, et al. Vertebral artery occlusion after acute cervical spine trauma. Spine (Phila Pa 1976) 2000; 25: 1171-1177.

25. Arnold M, Cumurciuc R, Stapf C, et al. Pain as the only symptom of cervical artery dissection. J Neurol Neurosurg Psychiatry 2006; 77: 1021-1024.

26. Herrera DA, Vargas SA, Dublin AB. Endovascular treatment of traumatic injuries of the vertebral artery. AJNR Am J Neuroradiol 2008; 29: 1585-1589.

27. Wang H, Orbach DB. Traumatic dissecting aneurysm at the vertebrobasilar junction in a 3-month-old infant: evaluation and treatment strategies. Case report. J Neurosurg Pediatr 2008; 1: 415-419.
28. Gottesman RF, Sharma P, Robinson KA, et al. Imaging characteristics of symptomatic vertebral artery dissection: a systematic review. Neurologist 2012; 18: 255-260.

29. Provenzale JM, Sarikaya B, Hacein-Bey L, Wintermark M. Causes of misinterpretation of cross-sectional imaging studies for dissection of the craniocervical arteries. AJR Am J Roentgenol 2011; 196: 45-52.

30. Kitanaka C, Tanaka J, Kuwahara M, Teraoka A. Magnetic resonance imaging study of intracranial vertebrobasilar artery dissections. Stroke 1994; 25: 571-575.

31. Mascalchi M, Bianchi MC, Mangiafico S, et al. MRI and MR angiography of vertebral artery dissection. Neuroradiology 1997; 39: 329-340.

32. Leclerc X, Lucas C, Godefroy O, et al. Preliminary experience using contrast-enhanced MR angiography to assess vertebral artery structure for the follow-up of suspected dissection. AJNR Am J Neuroradiol 1999; 20: 1482-1490.

33. Huston J 3rd, Bernstein MA, Riederer SJ. Feathering: Vertebral artery pseudostenosis with elliptical centric contrast-enhanced MR angiography. AJNR Am J Neuroradiol 2006; 27: 850-852.

34. Levy C, Laissy JP, Raveau V, et al. Carotid and vertebral artery dissections: three-dimensional time-of-flight MR angiography and MR imaging versus conventional angiography. Radiology 1994; 190: 97-103.

35. Miaux Y, Cognard C, Martin-Duverneuil N, et al. Flow-related enhancement in the vertebral plexus mimicking an intramural hematoma. AJNR Am J Neuroradiol 1996; 17: 191-192.

36. Schievink WI, Piepgras DG. Cervical vertebral artery aneurysms and arteriovenous fistulae in neurofibromatosis type 1: case reports. Neurosurgery 1991; 29: 760-765.

37. Houser OW, Mokri B, Sundt TM Jr., et al. Spontaneous cervical cephalic arterial dissection and its residuum: angiographic spectrum. AJNR Am J Neuroradiol 1984; 5: 27-34.

38. Ozdoba C, Sturzenegger M, Schroth G. Internal carotid artery dissection: MR imaging features and clinical-radiologic correlation. Radiology 1996; 199: 191-198.

39. Bartels E, Flugel KA. Evaluation of extracranial vertebral artery dissection with duplex color-flow imaging. Stroke 1996; 27: 290-295.

40. Sinnathamby M, Rao SV, Weber DG. Increased detection of blunt carotid and vertebral artery injury after implementation of diagnostic imaging pathway in level 1 trauma centre in Western Australia. Injury 2017; 48: 1917-1921.

41. Cox MW, Whittaker DR, Martinez C, et al. Traumatic pseudoaneurysms of the head and neck: early endovascular intervention. J Vasc Surg 2007; 46: 1227-1233.

42. CADISS trial investigators, Markus HS, Hayter E, et al. Antiplatelet treatment compared with anticoagulation treatment for cervical artery dissection (CADISS): a randomised trial. Lancet Neurol 2015; 14: 361-367.

43. Spanos K, Karathanos C, Stamoulis K, Giannoukas AD. Endovascular treatment of traumatic internal carotid artery pseudoaneurysm. Injury 2016; 47: 307-312. 